Meta

Journal des traducteurs

Translators' Journal

\title{
Lexique anglais-français de neurochirurgie
}

\section{Michèle Valiquette}

Volume 36, numéro 4, décembre 1991

URI : https://id.erudit.org/iderudit/004522ar

DOI : https://doi.org/10.7202/004522ar

Aller au sommaire du numéro

Éditeur(s)

Les Presses de l'Université de Montréal

ISSN

0026-0452 (imprimé)

1492-1421 (numérique)

Découvrir la revue

Citer cet article

Valiquette, M. (1991). Lexique anglais-français de neurochirurgie. Meta, 36(4), 633-644. https://doi.org/10.7202/004522ar d'utilisation que vous pouvez consulter en ligne.

https://apropos.erudit.org/fr/usagers/politique-dutilisation/ 


\section{ÉTUDES TERMINOLOGIQUES ET LINGUISTIQUES}

\section{LEXIQUE ANGLAIS-FRANÇAIS DE NEUROCHIRURGIE}

Si l'acte le plus élémentaire de «chirurgie crânienne», la trépanation, est vieux comme l'humanité - on a retrouvé des crânes préhistoriques avec des brèches osseuses faites par l'homme - la neurochirurgie proprement dite est récente. Ses premières tentatives, d'ailleurs isolées et sans aucune coordination, remontent seulement à la fin du XIX ${ }^{\mathrm{e}}$ siècle ou au début du nôtre, mais il faudra attendre les vingt années qui suivent la guerre de 19141918 pour qu'elle soit reconnue dans le monde entier comme une spécialité nouvelle, entièrement originale et très différente de la chirurgie générale. Le mot neurochirurgie ne figure même pas dans la première édition du Larousse du XX $X^{e}$ siècle, publiée entre 1928 et 19331.

Reposant sur un ensemble de connaissances anatomiques, neurologiques et biologiques du corps humain, la neurochirurgie a pour objet l'étude des lésions du système nerveux et leur traitement par voie chirurgicale et par antibiothérapie.

Située aux confluents de la chirurgie et de l'anatomie du système nerveux, la neurochirurgie possède une terminologie qui lui est propre tant en langue anglaise qu'en langue française. Toutefois nos recherches nous ont amenés à constater l'absence d'un vocabulaire bilingue spécialisé en la matière.

Notre document se présente sous la forme d'un lexique anglais-français de neurochirurgie qui compte 203 notions, désignées par des termes anglais et leurs équivalents français classés selon l'ordre alphabétique.

Les notions traitées appartiennent à cinq sous-domaines: généralités, anatomie du système nerveux, examens radiologiques, instruments chirurgicaux et techniques chirurgicales. Afin d'éviter une pléthore de synonymes, seuls ont été retenus les termes provenant des ouvrages lexicographiques médicaux qui ont pu être attestés dans la documentation du domaine dépouillée.

A été exclue de ce document la terminologie des traumatismes crâniens qui a fait l'objet du mémoire de traduction intitulé French-English Glossary of Disorders of the Central Nervous System, présenté à la Faculté des études supérieures de 1'Université de Montréal en 1986.

Au terme de notre recherche, nous espérons que le Lexique anglais-français de neurochirurgie ci-joint permettra de combler certains besoins d'expression des spécialistes tant de la langue (rédacteurs, traducteurs, terminologues et interprètes) que du domaine (praticiens et étudiants en neurochirurgie). 
LEXIQUE ANGLAIS-FRANÇAIS DE NEUROCHIRURGIE

1. anesthesia anaesthesia

2. angiography

3. anterior rhizotomy

4. approach

5. arachnoid

6. artery arteria

7. aspiration

8. audiometry

9. auditory evoked response

brain stem auditory evoked potentials acoustic evoked brain stem potentials auditory evoked responses auditory evoked potentials BAEPs

10. balloon catheter

11. biopsy

12. bipolar coagulator bipolar electrocoagulator bipolar coagulator unit bipolar unit

13. brain

14. brain scan

15. brain stem brainstem

16. burr hole burrhole exploratory burr hole

17. cannula canula

18. capsula capsule

19. carotid angiography carotid arteriography carotid imaging anesthésie

angiographie

radicotomie antérieure rhizotomie antérieure

voie d'abord

abord

abord chirurgical

arachnoïde

méninge arachnoïdienne

artère

aspiration

audiométrie

méthode des potentiels évoqués auditifs

potentiels évoqués auditifs PEA

cathéter à ballonnet sonde à ballonnet

biopsie

pince bipolaire pince coagulante bipolaire pince à coagulation bipolaire pince porte-courant bipolaire encéphale scanner cérébral scanner crânien

tronc cérébral

trou de fraise

trou de trépan explorateur

canule

sonde cannelée

capsule

angiographie carotidienne artériographie carotidienne 
20. catheter

21. cerebellopontine angle cerebellopontile angle ponto-cerebellar angle

22. cerebellum

23. cerebral angiography

24. cerebral cortex

25. cerebral hemisphere

26. cerebrospinal fluid CSF

27. cerebrum

28. cingulectomy

29. cingulotomy

30. cisternal puncture

31. cisternography

32. clamp

33. clip

34. $\mathrm{CO}_{2}$ laser

35. coagulation

36. coagulator

37. commissurotomy

38. contrast medium

39. cordotomy chordotomy

40. corpus callosum

41. cortectomy

42. cortex

43. corticography

44. cranial nerves

45. craniectomy

46. craniotome

47. craniotomy

48. curette curet

49. diploe cathéter

angle ponto-cérébelleux

cervelet

angiographie cérébrale artériographie cérébrale

cortex cérébral

hémisphère cérébral

liquide céphalorachidien

liquide céphalo-rachidien

LCR

L.C.-R.

cerveau

cingulectomie

cingulotomie

ponction sous-occipitale cisternographie

clamp

clip

laser $\mathrm{CO}_{2}$

laser $\mathrm{CO}_{2}$

neurochirurgical

coagulation

pince coagulante

commissurotomie

substances de contraste moyen de contraste produit de contraste

cordotomie

chordotomie

corps calleux

cortectomie

cortex

corticographie

nerfs crâniens

craniectomie

craniotome

craniotomie

curette

diploé 
50. discography diskography

51. drain

52. drainage surgical drainage

53. drapes

54. dura mater dura

55. echoencephalography echo-encephalography

56. echography

57. electrocoagulation

58. electrocorticography electro-corticography ECoG

59. electroencephalography EEG

60. electromyography EMG

61. electronystagmography ENG

62. electroretinography ERG

63. embolization

64. encephalography

65. endarterectomy

66. evoked cortical potential's evoked cortical potentials

67. excision exsection

68. exeresis

69. eyeglasses

70. eyepiece

71. falx

72. fissure of Sylvius Sylvian fissure

73. Fogarty catheter

74. foramen magnum discographie

diskographie

drain

drainage

drainage neurochirurgical

champs

dure-mère

méninge dure

echoencéphalographie

écho-encéphalographie

échographie

électrocoagulation

électro-coagulation

électrocorticographie

électro-corticographie

ECoG

électroencéphalographie

électro-encéphalographie

EEG

électromyographie

examen électromyographique

EMG

lectronystagmographie

ENG

électrorétinographie

E.R.G.

embolisation

encéphalographie

endartériectomie

méthode des potentiels évoqués

potentiel évoqué

excision

exérèse

lunettes

oculaire

faux

scissure de Sylvius

cathéter de Fogarty

sonde type Fogarty

foramen magnum

trou occipital 
75. foramen of Monro

76. forceps

77. fractional encephalography

78. frontal approach

79. frontal lobe

80. frontal lobectomy

81. gamma encephalography

82. general anesthesia

83. Gigli saw Gigli's wire saw

84. gray matter

85. headholder head fixation device head rest

86. hemilanectomy

87. hemispherectomy

88. hemoclip

89. hemostasis

90. hook

91. horizontal position hyperthermia

92. hypophysectomy hypophysiectomy

93. hypothermia

94. incision operative incision

95. indwelling catheter

96. internal carotid artery

97. intracranial pressure ICP

98. iodoventriculography

99. laminagraphy laminography

100. laminectomy

101. laryngoscopy

102. lateral position lateral decubitus position

103. lens trou de Monro

canal de Monro

orifice interventriculaire

pince

encéphalographie fractionnée

voie frontale

lobe frontal

lobectomie frontale

gamma-encéphalographie

anesthésie générale

scie de Gigli

fil-scie de Gigli

substance grise

appui-tête

hémilanectomie

hémisphérectomie

hémoclip

hémostase

hémostasie

crochet

décubitus horizontal

hyperthermie

hypophysectomie

hypothermie

incision

sonde à demeure

artère carotide interne

pression intracrânienne

iodoventriculographie iodo-ventriculographie

laminagraphie

laminectomie

laryngoscopie

décubitus latéral position latérale

lentille 
104. leucotomy leukotomy frontal leukotomy frontal lobotomy

105. ligature

106. line of incision

107. lobe

108. lobectomy

109. lobotomy

110. local anesthesia

111. loupe magnifting loupe operating loupe power loupe

112. lumbar puncture spinal tap

113. magnetic resonance imaging MR imaging MRI

114. magnification microscope magnification

115. Mayo scissors

116. Metzembaum scissors

117. microneurosurgery microneurosurgical technique

118. microsurgery micro-operative procedure micro-operative technique

119. monitoring

120. myelography

121. myelotomy

122. Nd:Yag laser

123. needle holder

124. nervous system

125. neurectomy

126. neurosurgeon neurological surgeon leucotomie

leucotomie préfrontale

lobotomie frontale

lobotomie préfrontale

ligature

ligne d'incision

lobe

lobectomie

lobotomie

anesthésie locale

loupe

ponction lombaire

PL

imagerie par résonance magnétique IRM

magnification

ciseaux Mayo

ciseaux de Mayo

ciseaux Metzembaum

ciseaux de Metzembaum

microneurochirurgie

microchirurgie

surveillance électronique monitorage monitoring

myélographie

myélotomie

laser Nd-Yag

laser Néodinium-Yag

Nd-Yag

porte-aiguille

système nerveux

neurectomie

névrotomie

neurochirurgien 
127. neurosurgery

neurological surgery

neurosurgical approach

neurosurgical operation

neurosurgical procedure

neurosurgical technique

128. nuclear magnetic resonance

Nuclear Magnetic Resonance NMR

129. nystagmography

130. occipital lobe

131. occipital lobectomy

132. operating microscope dissecting microscope operative microscope surgical microscope

133. operating room

neurochirurgie

134. operating table

135. operation

136. operating field operating site operative field operative site operation field surgical field

137. operator operating surgeon

138. parietal lobe

139. periosteum periost

140. phlebography venography

141. pia mater pia

142. pneumoencephalograhy pneumencephalography air encephalography

143. position

144. positron emission tomography Positron Emission Tomography PET scanning

145. posterior approach posterior cranial fossa operation posterior fossa exploration posterior fossa operation

résonance magnétique nucléaire $\mathrm{RMN}$

nystagmographie

lobe occipital

lobectomie occipitale

microscope opératoire

salle d'opération

table d'opération

opération

champ opératoire région opératoire

opérateur

lobe pariétal

périoste

phlébographie veinographie pie-mère méninge molle pneumo-encéphalographie encéphalographie gazeuse

position

scanner à positron

voie postérieure 
posterior fossa procedure posterior fossa surgery

146. posterior rhizotomy dorsal rhizotomy

147. probe

148. prone position prone

149. psychosurgery

150. puncture pure tone audiometry

151. resection

152. retractor brain retractor

153. rhizotomy radicotomy

154. scalp

155. scanography

156. scintigraphy

157. scissors

158. sella turcica sella sellar region

159. sinus

160. sitting position

161. skin incision

162. skull

163. somatensory evoked potentials somatosensory evoked responses SEPs

164. speech audiometry

165. stereotaxic frame

166. stereotaxis stereotaxic approach

stereotaxic surgery

167. subfrontal approach subfrontal route

168 suboccipital approach suboccipital operation suboccipital route radicotomie postérieure rhizotomie postérieure

sonde

décubitus ventral position ventrale

psychochirurgie psycho-chirurgie intervention psychochirurgicale ponction audiométrie tonale résection

écarteur

rhizotomie radicotomie cuir chevelu scanographie scintigraphie ciseaux selle turcique

sinus

position assise

incision cutanée crâne

méthode des potentiels évoqués sensitifs potentiels évoqués somesthésiques PES

audiométrie vocale cadre stéréotaxique neurochirurgie stéréotaxique démarche stéréotaxique intervention stéréotaxique chirurgie stéréotaxique opération stéréotaxique abord sous-frontal exploration sous-frontale abord sous-occipital voie sous-occipitale 
169. subtemporal approach

subtemporal route

170. sucker

suction apparatus

suction device

suction unit

171. supine position supine

172. supratentorial exploration supratentorial surgery

173. surgeon

174. surgery

175. suture

176. sympathectomy sympathetectomy sympathicectomy

177. temporal approach

178. temporal lobe

179. temporal lobectomy

180. thalamotomy

181. tomography

182. topectomy

183. tracheotomy

184. tractomy

185. transection

186. translabyrinthine approach translabyrinthine operation translabyrinthine route

187. transsphenoidal approach trans-sphenoidal approach transsphenoidal microsurgery transsphenoidal operation trans-sphenoidal operation transsphenoidal procedure transsphenoidal route transsphenoidal surgical procedure transsphenoidal surgery

188. trepanation

189. trephination trephine opening

190. trephine

191. unipolar coagulator unipolar type of coagulator abord sous-temporal

exploration sous-temporale voie sous-temporale

suceur

décubitus dorsal

position dorsale

voie sus-tentorielle

chirurgien

chirurgie

suture

sympathectomie

sympathicectomie

voie temporale

lobe temporal

lobectomie temporale

thalamotomie

tomographie

topectomie

trachéotomie

tractomie

transsection

abord translabyrinthique

abord transsphénoïdal

voie transsphénoïdale

voie d'abord transsphénoïdale

trépanation

tréphination

technique du disque de tréphine

tréphine

pince monopolaire 
192. vascularization

193. vein

194. ventricular drainage

195. ventricular puncture

196. ventriculocisternostomy ventriculo-cisternostomy Torkildsen's operation

Torkildsen's ventriculo-cisternostomy

197. ventriculography

198. ventriculostomy

199. vertebral angiography

200. visual evoked potential visual evoked potentials evoked visual potential VEPs

201. white matter

202. xanthochromia

203. zygoma vascularisation

veine

drainage ventriculaire

ponction ventriculaire

ventriculocisternostomie ventriculostomie de Torkildsen ventriculocysternostomie de

Torkildsen

ventriculographie ventriculostomie angiographie vertébrale artériographie vertébrale méthode des potentiels évoqués visuels potentiels évoqués visuels PEV

substance blanche xanthochromie

zygoma

MichÈle VAliQueTte Secrétariat d'État, Montréal, Canada

NOTE

1. Marcel David et Paul Guilly, La Neurochirurgie, Que sais-je?, Paris, PUF, p. 5.

\section{BIBLIOGRAPHIE}

Monographies

ADAM, Yves (1985): Neurochirurgie du praticien, Paris, Maloine, $147 \mathrm{p}$.

ALLEN, Marshall B., Gernot S. DOESTCH, R. Arthur GINDIN, Floyd L. HAAR and F. YAGHMAI (1978): A Manual of Neurosurgery, Baltimore, London, Tokyo, University Park Press, $282 \mathrm{p}$.

CAMBIER, J., H. DEHEN, J. POIRIER et J.-L. RIBADEAU DUMAS (1976): Propédeutique neurologique, Paris, New York, Barcelone, Milan, Masson, 193 p.

DAVID, Marcel, Henri POURPRE, Jean LEPOIRE et Domenico DILENGE (1961): Neurochirurgie, Paris, Éditions médicales Flammarion, 1961, XXIII + 973 p.

DAVID, Marcel et Paul GUILLY (1970): La Neurochirurgie, Paris, Que sais-je ?, PUF, $\mathrm{n}^{\circ} 1369,124 \mathrm{p}$

GUIBERT, André et Claude GUIBERT (1973): Schémas d'anatomie du système nerveux central — Les nerfs crâniens: étude anatomo clinique, $2^{e}$ éd., Paris, Maloine, 147 p.

HAYWARD, Richard (1980): Essentials of Neurosurgery, Ox Ford, London, Melbourne, Edinburgh, Blackwell Scientific Publications, VII $+275 \mathrm{p}$

HAZEMAN, P. and M. MASSON (1987): Électro-encéphalographie, Paris, New York, Barcelone, Milan, Mexico, Sao Paolo, Masson, $96 \mathrm{p}$.

HOWES, James R. (1977) : Patient Care in Neurosurgery, Boston, Little Brown and Co., 228 p.

JENNETT, W. Bryan (1970): An Introduction to Neurosurgery, 2nd Ed., Saint-Louis, The C.V. Mosby Co., 365 p.

LANDOLT, A.M. (Series Ed.) (1984): Complications in Neurosurgery I, Basel, Munchen, Paris, London, New York, Tokyo, Sydney, Karyer, 171 p. (Series: Progress in Neurological Surgery, Volume 11).

LITEL, Gerard R. (1980): Neurosurgery and the Clinical Team - A Guide for Nurses, Technicians, and Students, with contributions by Patricia E. MAHONEY and Joyce KEEN, New York, Springer Publishing Company, $306 \mathrm{p}$. 
MULLAN, Sean (1961): Essentials of Neurosurgery for students and practitioners, New York, Springer Publishing Company, $273 \mathrm{p}$.

NETTER, Frank H. (1972): A Compilation of paintings of the normal and pathologic anatomy of the nervous system, New York, CIBA Collection of Medical Illustrations, $168 \mathrm{p}$.

O'BRIEN, Mark S. (Ed.) (1978): Pediatric Neurological Surgery, New York, Raven Press, 206 p. (Seminars in Neurological Surgery).

PATEL, Jean et Lucien LÉGER (dir) (1975): Nowveau traité de technique chirurgicale, Paris, Masson et Cie, $631 \mathrm{p}$.

RASMUSSEN, Theodore and Paul MARINO (Eds.) (1979): Functional Neurosurgery, New York, Raven Press, $\mathrm{X}+278 \mathrm{p}$.

ROUX, François-Xavier (1986) : Le Laser CO² neurochirurgical, Paris, Maloine S.A, 85 p.

SMITH, Bernard H. (1979): Differential Diagnosis Neurology, NewYork, Arco Publishing, Inc., 312 p., ARCO Diagnosis Series.

SMITH, Robert R. (1980) : Essentials of Neurosurgery, Philadelphia, Toronto, J.B. Lippincot Company, 321 p.

TILLIER, Jean-Noël et Didier PIERRON (1985): Neurologie-Neurochirurgie, Paris, Maloine S.A., 186 p., Coll. Dossiers médico-chirurgicaux, Fasicule 27.

WEINER, Howard L. and Lawrence P. LEVITT (1983): Neurology for the House Officer, 3th Ed., Baltimore/ London, Williams, $204 \mathrm{p}$.

WISE, Burton L. ( 1978): Preoperative and Postoperative Care in Neurosurgical Surgery, 2nd Ed., Springfield, Illinois, Charles $\mathrm{C}$. Thomas, $187 \mathrm{p}$.

YASARGIL, M.G. et al. (1969): Microsurgery Applied to Neurosurgery, 2nd Ed., Stuttgart, Georg Thieme Verlag, New York and London, Academic Press, $230 \mathrm{p}$.

YASARGIL, M.G., coll. R.D. SMITH, P.H. YOUNG and P.L. TEDDY (1984): Microsurgery in 4 Volumes. I. Microsurgical Anatomy of the Basal Cisterns and Vessels of the Brain, Diagnostic Studies, General Operative Techniques and Pathological Considerations of the Intracranial Aneurysms, Stuttgart, Georg Thieme Verlag, $371 \mathrm{p}$.

YASARGLL, M.G., coll. R.D. SMITH, P.H. YOUNG and P.L. TEDDY (1984): Microsurgery in 4 Volumes. II. Clinical Considerations, Surgery of the Intracranial Aneurysms and Results, Stuttgart, Georg Thieme Verlag, $386 \mathrm{p}$.

YOUSMA,S, Julian, R. (Ed.) (1982): Neurological Surgery, Vol. I. A Comprehensive Reference Guide to the Diagnosis and Management of Neurosurgical Problems, 2nd Ed., Philadephia, London, Toronto, Mexico City, Rio de Janeiro, Sydney, Tokyo, W.B. Saunders Company, 635 p.

YOUSMANS, Julian, R. (Ed.) (1982): Neurological Surgery. Volume 2. A Comprehensive Reference Guide to the Diagnosis and Management of Neurosurgical Problems, 2nd Ed., Philadephia, London, Toronto, Mexico City, Rio de Janeiro, Sydney, Tokyo, W.B. Saunders Company, 564 p.

Actes

CARREA, Raul and FLENI Computed Tomography Center (Eds.) David LEVAY, (co-ed.) (1978): Neurological Surgery with emphasis on non-invasive methods of diagnosis and treatment, Amsterdam, Oxford, Excerpta, Medica, XIV $+420 \mathrm{p}$. (Proceedings of the Sixth International Congress of Neurological Surgery, Sao Paolo, June $19-25,1977$ ).

SCHMIDEK, Henry H. and William H, SWEET (1976): Current Techniques in Operative Neurosurgery, New York, Grunne \& Stratton, Inc., XIII + 517 p. (Habermann Symposium on Current Techniques in Operative Neurosurgery, New York, 1976).

WALTER, W., M. BRANDT, M. BROCK and M. KLINGER (Eds.) (1988): Modern Methods in Neurosurgery, Berlin, Heidelberg, New York, London, Paris, Tokyo, Springer-Verlag, 303 p. (Proceedings of the 38th Annual Meeting of the Deutsche Gesellschaft fur Neurochirurgie, Munster, May 3-6, 1987).

Ouvrages lexicographiques

CRITCHLEY, Macdonald (Ed.-in-Chief) (1978): Butterworths Medical Dictionary, 2nd Ed., London, Boston, Sydney, Wellington, Durban, Toronto, Butterworths, XXXII + $1942 \mathrm{p}$

DOMART, professeur A. et docteur J. BOURNEUF (dir) (1988): Nouveau Larousse médical, Paris, Larousse, $1142 \mathrm{p}$.

DORLAND'S Illustrated Medical Dictionary (1988): 27th Ed., Philadelphia, London, Toronto, Montréal, Sydney, Tokyo, XXXII + $1887 \mathrm{p}$.

GARNIER, Marcel, Valery DELAMARE, Jean DELAMARE, Thérèse DELAMARE-RICHE et Jacques DELAMARE, Coll. François DELAMARE et Élisabeth GÉLIS-MALVILLE (1989): Dictionnaire des termes de médecine, $22^{\circ}$ édition, Paris, Maloine, XIII + $1031 \mathrm{p}$.

MANUILA, A. L. MANUILA, M. NICOLE et H. LAMBERT, Coll. J. HUREAU et J. POLONOVSKI (1970): Dictionnaire français de médecine et biologie en quatre volumes, Quatre tomes, Paris, Masson \& Cie, $3542 \mathrm{p}$. 
MANUILA, A, L. MANUILA, M. NICOLE et H. LAMBERT, Coll. J. HUREAU et J. POLONOVSKI (1981) :

Dictionnaire français de médecine et biologie - Supplément, Paris, Masson \& Cie, $192 \mathrm{p}$.

International Dictionary of Medicine and Biology in Three Volumes (1986): New York, Chichester, Brisbane, Toronto, Singapore, John Wiley \& Sons, LI + 3200 p. (A Wiley MedicalPublication).

Dictionnaire de médecine (1982): Paris, Médecine-Sciences, Flammarion, $935 \mathrm{p}$.

Illustrated Stedman's Medical Dictionary (1982): 24th Ed., Baltimore, London, Williams \& Wilkins, XLVII + $1678 \mathrm{p}$.

Taber's Cyclopedic Medical Dictionary (1985): Ed. 15 Illustrated, Philadelphia, F.A. Davis Company, 2170 p.

\section{Ouvrages encyclopédiques}

BOUCHE, P. (1983): «Électromyographie clinique. Apport de la sémiologie électrique dans l'examen des maladies neuromusculaires», Encyclopédie médico-chirurgicale. Neurologie, vol. 2, Paris, Encyclopédie médico-chirurgicale, $17030 \mathrm{~B}^{10}, 14 \mathrm{p}$.

CHEVALERAUD, J. (1982): «Explorations électrophysiologiques sensorielles», Encyclopédie médicochirurgicale. Ophtalmologie, vol. 2, Paris, Encyclopédie médico-chirurgicale, $21046 \mathrm{~A}^{10}, 10 \mathrm{p}$.

CHIRAS, J. et J. BORIES (1984): «L'angiographie cérébrale normale», Encyclopédie médico-chirurgicale. Neurologie, vol. 2, Paris, Encyclopédie médico-chirurgicale, 1984, 17032 D $10,20 \mathrm{p}$.

GASTAUT, Henri (1980): «L'électroencéphalographie clinique en neurologie», Encyclopédie médicochirurgicale. Neurologie, vol. 2, Paris, Encyclopédie médico-chirurgicale, 17031 A ${ }^{10}, 6$ p.

Grand Dictionnaire encyclopédique Larousse (1982-1985): Paris, Librairie Larousse, 10 volumes.

HALSTED, James A. and Charles H. (1981): The Laboratory in Clinical Medicine - Interpretation and Application, 2nd Ed., Philadephia, London, Toronto, Sydney, Tokyo, W.B. Saunders Company, XXXV + $1083 \mathrm{p}$.

KERAVEL, Y., J.-P, N. N'GUYEN et P. CESARO (1977): «Vues anatomiques du cortex cérébral», Encyclopédie médico-chirurgicale. Neurologie, vol. 1, Paris, Encyclopédie médico-chirurgicale, $17001 \mathrm{~J} 10$, $26 \mathrm{p}$.

MAUGUIERE, F. et C. FISHER (1990): «Les potentiels évoqués en neurologie», Encyclopédie médicochirurgicale. Neurologie, vol, 2, Paris, Encyclopédie médico-chirurgicale, $17031 \mathrm{~B}^{10}, 26 \mathrm{p}$.

RAPIN, Maurice (1986): Le Grand Dictionnaire encyclopédique médical, Paris, Médecine-Sciences Flammarion, XXIII + $1394 \mathrm{p}$.

\section{Norme}

Association française de normalisation (1987): «Matériel biomédical», t. 2, Matériel pour la chirurgie et pour les handicapés, $3^{\mathrm{e}}$ éd., Paris, AFNOR, 443 p. (Recueil de normes françaises).

Périodique

Les travaux du Comité d'étude des termes médicaux français (1989): L'Actualité terminologique, Secrétariat d'État, Langues officielles et Traduction, Ottawa, 22-1, pp 5-10. 